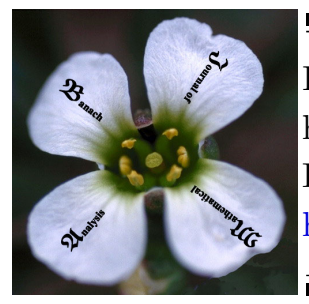

Banach J. Math. Anal. 9 (2015), no. 3, 173-193

http://doi.org/10.15352/bjma/09-3-13

ISSN: $1735-8787$ (electronic)

http://projecteuclid.org/bjma

\title{
APPROXIMATE UNITARY EQUIVALENCE OF NORMALOID TYPE OPERATORS
}

\author{
SEN ZHU
}

Communicated by P. Y. Wu

\begin{abstract}
In this paper, we explore approximate unitary equivalence of normaloid operators and classify several normaloid type operators including transaloid operators, polynomial-normaloid operators and von Neumann operators up to approximate unitary equivalence. As an application, we explore approximation of transaloid operators with closed numerical ranges. Among other things, it is proved that those transaloid operators with closed numerical ranges are norm dense in the class of transaloid operators.
\end{abstract}

\section{INTRODUCTION AND PRELIMINARIES}

Throughout this paper, $\mathcal{H}$ will always denote a complex separable Hilbert space endowed with the inner product $\langle\cdot, \cdot\rangle$. We let $\mathcal{B}(\mathcal{H})$ denote the algebra of all bounded linear operators on $\mathcal{H}$. In this paper, an operator will always mean a bounded linear operator.

Let $T \in \mathcal{B}(\mathcal{H})$. We denote by $\sigma(T)$ the spectrum of $T$, and by $\gamma(T)$ the spectral radius of $T$, that is, $\gamma(T)=\max \{|\lambda|: \lambda \in \sigma(T)\}$. Recall that $T$ is said to be normaloid if $\|T\|=\gamma(T)$, and $T$ is said to be transaloid if $T-\lambda$ is normaloid for all $\lambda \in \mathbb{C}$.

The notion of normaloid operators was introduced by Wintner [20] in terms of numerical range. The numerical range of $T$ is the nonempty set

$$
W(T) \triangleq\{\langle T \xi, \xi\rangle: \xi \in \mathcal{H},\|\xi\|=1\} .
$$

Date: Received: Jun. 7, 2014; Accepted: Nov. 4, 2014.

2010 Mathematics Subject Classification. Primary 47B20; Secondary 47A25, 47A12.

Key words and phrases. Normaloid operators, transaloid operators, von Neumann operators, approximate unitary equivalence, numerical range. 
The numerical radius of $T \in \mathcal{B}(\mathcal{H})$ is

$$
w(T) \triangleq \sup \{|z|: z \in W(T)\} .
$$

An operator $T$ is normaloid if and only if $w(T)=\|T\|$ (see [9, Prob. 218]).

The most obvious examples of transaloid operators are hyponormal operators, which have been extensively studied by many authors. When $T$ is hyponormal (that is, $T^{*} T-T T^{*} \geq 0$ ), it is well known that $T-\lambda$ is hyponormal and hence $\gamma(T-\lambda)=\|T-\lambda\|$ for all $\lambda \in \mathbb{C}$. So each hyponormal operator is transaloid. Thus the notion of transaloidity can be viewed as a generalization of hyponormality, and has been studied by many authors [1, 6, 8, 12, 13, 15, 21].

There are two other related classes of operators. We say that an operator $T \in \mathcal{B}(\mathcal{H})$ is polynomial-normaloid (p-normaloid for short) if $p(T)$ is normaloid for each polynomial $p(\cdot)([2])$. Furthermore, if $f(T)$ is normaloid for each rational function $f$ with poles off $\sigma(T)$, then $T$ is called a von Neumann operator ([5, Def. 9.1]). The notion of von Neumann operators is closely related to a notion of spectral sets due to von Neumann [16]. Each subnormal operator is a von Neumann operator ([5, Prop. 9.2]).

The main aim of this note is to classify normaloid operators, transaloid operators, p-normaloid operators and von Neumann operators up to approximate unitary equivalence. Recall that two operators $A, B \in \mathcal{B}(\mathcal{H})$ are said to be approximately unitarily equivalent, denoted by $A \cong{ }_{a} B$, if there exist unitary operators $\left\{U_{n}\right\}_{i=1}^{\infty}$ such that $A U_{n}-U_{n} B \rightarrow 0$ as $n \rightarrow \infty$, or equivalently, the closures of the unitary equivalence classes of $A$ and $B$ coincide. When two operators are approximately unitarily equivalent, they have many common properties. In particular, all the above-mentioned normaloid type properties are invariant under approximate unitary equivalence.

To state our main results, we first introduce some notation and terminology.

Given a convex subset $\Gamma$ of $\mathbb{C}$, we denote by $\mathcal{E}(\Gamma)$ the set of extreme points of $\Gamma$. Given a compact subset $\sigma$ of $\mathbb{C}$, the polynomially convex hull of $\sigma$, denoted by $\widehat{\sigma}$, is defined to be the set of all points $w$ such that for every polynomial $p$

$$
|p(w)| \leq \max \{|p(z)|: z \in \sigma\} .
$$

By the Maximum Modulus Theorem (MMT for short) for analytic functions, $\widehat{\sigma}$ is obtained from $\sigma$ by filling any "holes" that may exist in $\sigma$. Then one can deduce that $\widehat{\sigma}$ equals the complement of the unbounded component of $\mathbb{C} \backslash \sigma$.

The main results of this paper are listed as follows.

Theorem 1.1. Let $T \in \mathcal{B}(\mathcal{H})$. Then $T$ is normaloid if and only if $T$ is approximately unitarily equivalent to an operator of the form $N \oplus A$, where $N$ is normal and $\|A\| \leq\|N\|$; in addition, $N$ can be required to satisfy $\sigma(N)=\{z \in \sigma(T)$ : $|z|=\|T\|\}$.

In what follows, we write conv $\Gamma$ for the convex hull of a set $\Gamma \subseteq \mathbb{C}$, that is, the smallest convex set that includes $\Gamma$.

Theorem 1.2. An operator $T \in \mathcal{B}(\mathcal{H})$ is transaloid if and only if $T$ is approximately unitarily equivalent to an operator of the form $N \oplus A$, where $N$ is normal 
and $A$ satisfies

$$
\|A-\lambda\| \leq\|N-\lambda\|, \quad \forall \lambda \in \mathbb{C}
$$

in addition, $N$ can be required to satisfy $\sigma(N)=\mathcal{E}(\operatorname{conv} \sigma(T))$.

Theorem 1.3. An operator $T \in \mathcal{B}(\mathcal{H})$ is p-normaloid if and only if $T$ is approximately unitarily equivalent to an operator of the form $N \oplus A$, where $N$ is normal and $A$ satisfies

$$
\|p(A)\| \leq\|p(N)\|
$$

for any polynomial $p$; in addition, $N$ can be required to satisfy $\sigma(N)=\partial \widehat{\sigma(T)}$.

Theorem 1.4. An operator $T \in \mathcal{B}(\mathcal{H})$ is a von Neumann operator if and only if $T$ is approximately unitarily equivalent to an operator of the form $N \oplus A$, where $N$ is normal and $A$ satisfies

$$
\|f(A)\| \leq\|f(N)\|
$$

for any rational function $f$ with poles off $\sigma(T)$; in addition, $N$ can be required to satisfy $\sigma(N)=\partial \sigma(T)$.

Remark 1.5. By Theorems 1.1, 1.2, 1.3 and 1.4, each normaloid type property is determined up to approximate unitary equivalence by normal operators, and one can construct various examples of normaloid operators (see Example 4.5). As an application of main results, we shall prove that a unilateral weighted shift is normaloid if and only if $T$ is a von Neumann operator (Theorem 4.6).

The following result is an immediate consequence of our main results.

Corollary 1.6. Let $T \in \mathcal{B}(\mathcal{H})$ be normaloid. Then the $C^{*}$-algebra $C^{*}(T)$ generated by $T$ admits at least one character (that is, multiplicative linear functional) on it; in particular, if

(i) $\lambda \in \sigma(T)$ and $|\lambda|=\|T\|$, or

(ii) $T$ is transaloid and $\lambda \in \mathcal{E}(\operatorname{conv} \sigma(T))$, or

(iii) $T$ is p-normaloid and $\lambda \in \partial \widehat{\sigma(T)}$, or

(iv) $T$ is a von Neumann operator and $\lambda \in \partial \sigma(T)$,

then there exists a character $\varphi$ on $C^{*}(T)$ such that $\varphi(T)=\lambda$

Proof. Note that if an operator $T$ has the form $N \oplus A$ and $\lambda \in \sigma(N)$, where $N$ is normal, then the $C^{*}$-algebra $C^{*}(T)$ generated by $T$ admits a character $\varphi$ on it such that $\varphi(T)=\lambda$. Then, in view of Theorems 1.1,1.2,1.3 and 1.4, the desired result follows readily.

As an application of Theorem 1.2, we explore approximation of transaloid operators with closed numerical ranges.

The classical Toeplitz-Hausdorff theorem asserts that the numerical range of an operator is always convex $[18,10]$. Note that the numerical range of a finitedimensional operator is a continuous image of a compact set, and hence necessarily closed. However, this is not the case in infinite-dimensional space. For example, the numerical range of the unilateral shift on $l^{2}$ is the open unit disk. Since the unilateral shift is hyponormal and hence transaloid, this shows that a transaloid operator can have a non-closed numerical range. 
On the other hand, given an operator $T$, it is natural to explore the inclusion relationship between $\sigma(T)$ and $W(T)$. The convex hull of $\sigma(T)$ lies in the closure of $W(T)$ (see [17] or [9, Prob. 214]) and $W(T)^{-}$may be very much larger than conv $\sigma(T)$. So it is natural to ask when an operator $T$ satisfies $W(T)=\operatorname{conv} \sigma(T)$; in this case, $T$ has a closed numerical range.

We obtain the following result which asserts that those transaloid operators with closed numerical ranges are norm dense in the class of transaloid operators.

Theorem 1.7. Let $T \in \mathcal{B}(\mathcal{H})$ be transaloid. Then, given $\varepsilon>0$, there exists compact $K \in \mathcal{B}(\mathcal{H})$ with $\|K\|<\varepsilon$ such that $T+K$ is transaloid and $W(T+K)=$ conv $\sigma(T+K)$.

Recall that an operator $T$ is said to be convexoid if $W(T)^{-}=$conv $\sigma(T)$. Each transaloid operator is convexoid (see Lemma 2.8). Note that $W(T)=\operatorname{conv} \sigma(T)$ if and only if $T$ is a convexoid operator with closed numerical range.

Remark 1.8. Bourin [3, Prop. 1.3] proved that those operators with closed numerical ranges are norm dense in $\mathcal{B}(\mathcal{H})$. However, those operators $A \in \mathcal{B}(\mathcal{H})$ satisfying $W(A)=$ conv $\sigma(A)$ are not norm dense in $\mathcal{B}(\mathcal{H})$. Using the upper semi-continuity of spectrum and the continuity of numerical range [9, Prob. 220], one can check that the following operator on $\mathbb{C}^{2}$ is not a norm limit of convexoid operators:

$$
\left[\begin{array}{ll}
0 & 1 \\
0 & 0
\end{array}\right] .
$$

The following result describes when a transaloid operator is approximately unitarily equivalent to some operator with closed numerical range.

Theorem 1.9. A transaloid operator $T$ is approximately unitarily equivalent to some operator with closed numerical range if and only if conv $\sigma(T)$ has at most denumerable extreme points.

In this paper, we concentrate on complex separable infinite-dimensional Hilbert spaces. Our main results still hold for finite-dimensional Hilbert spaces, and the proofs follow similar lines.

The rest of this paper is organized as follows. In Section 2, we shall make some preparation. The proofs of Theorems 1.1 and 1.2 will be provided in Section 3. Section 4 is devoted to the proofs of Theorems 1.3 and 1.4. In the concluding section, we shall prove Theorems 1.7 and 1.9.

\section{Preparation}

For convenience, we first give some familiar notation and terminology.

Let $T \in \mathcal{B}(\mathcal{H})$. We denote by $\operatorname{ker} T$ and $\operatorname{ran} T$ the kernel of $T$ and the range of $T$ respectively. $T$ is called a semi-Fredholm operator, if ran $T$ is closed and either nul $T$ or nul $T^{*}$ is finite, where nul $T \triangleq \operatorname{dim} \operatorname{ker} T$ and nul $T^{*}=\operatorname{dim} \operatorname{ker} T^{*}$; in this case, ind $T \triangleq$ nul $T-$ nul $T^{*}$ is called the index of $T$. In particular, if $-\infty<$ ind $T<\infty$, then $T$ is called a Fredholm operator. The Wolf spectrum $\sigma_{\text {lre }}(T)$ and the essential spectrum $\sigma_{e}(T)$ are defined by

$$
\sigma_{\text {lre }}(T) \triangleq\{\lambda \in \mathbb{C}: T-\lambda \text { is not semi-Fredholm }\}
$$


and

$$
\sigma_{e}(T) \triangleq\{\lambda \in \mathbb{C}: T-\lambda \text { is not Fredholm }\}
$$

respectively.

If $\sigma$ is a clopen subset of $\sigma(T)$, then there exists an analytic Cauchy domain $\Omega$ such that $\sigma \subseteq \Omega$ and $[\sigma(T) \backslash \sigma] \cap \Omega^{-}=\emptyset$. We let $E(\sigma ; T)$ denote the Riesz idempotent of $T$ corresponding to $\sigma$, that is,

$$
E(\sigma ; T)=\frac{1}{2 \pi \mathrm{i}} \int_{\Gamma}(\lambda-T)^{-1} \mathrm{~d} \lambda,
$$

where $\Gamma=\partial \Omega$ is positively oriented with respect to $\Omega$ in the sense of complex variable theory. In this case, we denote $\mathcal{H}(\sigma ; T)=\operatorname{ran} E(\sigma ; T)$. If $\lambda$ is an isolated point of $\sigma(T)$, then $\{\lambda\}$ is a clopen subset of $\sigma(T)$ and we simply write $\mathcal{H}(\lambda ; T)$ instead of $\mathcal{H}(\{\lambda\} ; T)$; if, in addition, $\operatorname{dim} \mathcal{H}(\lambda ; T)<\infty$, then $\lambda$ is called a normal eigenvalue of $T$. Each normal eigenvalue of $T$ lies in the point spectrum $\sigma_{p}(T)$. The set of all normal eigenvalues of $T$ will be denoted by $\sigma_{0}(T)$. The reader is referred to [11, Chap. 1] or [4, page 210] for more about normal eigenvalues.

Lemma 2.1 ([4], page 366). Let $T \in \mathcal{B}(\mathcal{H})$. Then $\partial \sigma(T) \subseteq\left[\sigma_{0}(T) \cup \sigma_{\text {lre }}(T)\right]$.

Corollary 2.2. Let $T \in \mathcal{B}(\mathcal{H})$. Then $\sigma_{0}(T)$ is at most denumerable and each accumulation point of $\sigma_{0}(T)$ lies in $\sigma_{\text {lre }}(T)$.

The following lemma is clear.

Lemma 2.3. If $\sigma$ is a nonempty compact subset of $\mathbb{C}$, then conv $\sigma, \mathcal{E}(\operatorname{conv} \sigma)$ are both compact, $\mathcal{E}(\operatorname{conv} \sigma) \subseteq \partial \sigma$ and

$$
\max _{\mu \in \sigma}|\lambda-\mu|=\max _{\mu \in \operatorname{conv} \sigma}|\lambda-\mu|=\max \{|\lambda-\mu|: \mu \in \mathcal{E}(\operatorname{conv} \sigma)\}, \quad \forall \lambda \in \mathbb{C} .
$$

Throughout the following, we let $\mathcal{K}(\mathcal{H})$ denote the ideal of compact operators in $\mathcal{B}(\mathcal{H})$.

Definition 2.4. The essential numerical range of an operator $T \in \mathcal{B}(\mathcal{H})$ is the nonempty set

$$
W_{e}(T) \triangleq \bigcap_{K \in \mathcal{K}(\mathcal{H})} W(T+K)^{-}
$$

It is obvious that the essential numerical range is always compact and invariant under compact perturbations. The following result, due to Lancaster [14], describes the relationship between the numerical range and the essential numerical range of an operator.

Theorem 2.5 ([14]). If $A \in \mathcal{B}(\mathcal{H})$, then $W(A)^{-}=\operatorname{conv}\left(W(A) \cup W_{e}(A)\right)$.

As a consequence of Theorem 2.5, one can see that the numerical range is closed if and only if it contains the essential numerical range.

Lemma 2.6 ([7]). Let $T \in \mathcal{B}(\mathcal{H})$. Then $\lambda \in W_{e}(T)$ if and only if there exists an orthonormal sequence $\left\{e_{n}\right\}_{n=1}^{\infty}$ such that $\lim _{n}\left\langle T e_{n}, e_{n}\right\rangle=\lambda$.

Lemma 2.7. If $T \in \mathcal{B}(\mathcal{H})$, then $\sigma_{e}(T) \subseteq W_{e}(T)$. 
Proof. For any $K \in \mathcal{K}(\mathcal{H})$, it is clear that

$$
\sigma_{e}(T)=\sigma_{e}(T+K) \subseteq \sigma(T+K) \subseteq W(T+K)^{-} .
$$

It follows readily that $\sigma_{e}(T) \subseteq W_{e}(T)$.

Lemma 2.8. If $T \in \mathcal{B}(\mathcal{H})$ is transaloid, then $\operatorname{conv} \sigma(T)=W(T)^{-}$and $\|T\|=$ $\gamma(T)=w(T)$.

Proof. For a proof by contradiction, we assume that conv $\sigma(T) \neq W(T)^{-}$. Since it is obvious that $\sigma(T) \subseteq W(T)^{-}$, we have conv $\sigma(T) \subsetneq W(T)^{-}$. By the convexity of conv $\sigma(T)$ and $W(T)^{-}$, there exists $\mu \in \mathbb{C}$ such that

$$
\sup _{z \in W(T)}|\mu-z|>\sup _{z \in \sigma(T)}|\mu-z|,
$$

that is, $w(\mu-T)>\gamma(\mu-T)$. It follows that $\|\mu-T\|>\gamma(\mu-T)$, contradicting the fact that $T$ is transaloid. This completes the proof.

Lemma 2.9. If $T \in \mathcal{B}(\mathcal{H})$ is hyponormal, then $W_{e}(T)=\operatorname{conv} \sigma_{\text {lre }}(T)$.

Proof. Without loss of generality, we assume that $\sigma_{0}(T)=\left\{\lambda_{i}: i=1,2,3, \cdots\right\}$. Since $T$ is hyponormal, $T$ can be written as

$$
T=\left[\begin{array}{ccccc}
\lambda_{1} I_{1} & & & & \\
& \lambda_{2} I_{2} & & & \\
& & \lambda_{3} I_{3} & & \\
& & & \ddots & \\
& & & & A
\end{array}\right] \begin{gathered}
M_{1} \\
M_{2} \\
M_{3} \\
\vdots \\
M_{0}
\end{gathered}
$$

where $M_{i}=\operatorname{ker}\left(T-\lambda_{i}\right), I_{i}$ is the identity operator on $M_{i}$ for $i \geq 1$ and $M_{0}=$ $\left(\vee_{i \geq 1} M_{i}\right)^{\perp}$. Here $\vee$ denotes closed linear span. Obviously, $\operatorname{dim} M_{i}<\infty, A-\lambda_{i}$ is invertible for each $i \geq 1$ and it follows from Corollary 2.2 that $\operatorname{dist}\left(\lambda_{i}, \sigma_{\text {lre }}(T)\right) \rightarrow$ 0 . For each $i \geq 1$, we can find $\mu_{i} \in \sigma_{\text {lre }}(T)$ such that $\operatorname{dist}\left(\lambda_{i}, \sigma_{\text {lre }}(T)\right)=\left|\lambda_{i}-\mu_{i}\right|$. Then there exists a compact operator $K$ such that

$$
T+K=\left(\oplus_{i=1}^{\infty} \mu_{i} I_{i}\right) \oplus A .
$$

One can check that $\sigma_{0}(T+K)=\emptyset$ and it follows from Lemma 2.1 that $\partial \sigma(T+$ $K) \subseteq \sigma_{l r e}(T+K)$. Noting that $T+K$ is still hyponormal, we have

$$
\begin{aligned}
W(T+K)^{-} & =\operatorname{conv} \sigma(T+K)=\operatorname{conv} \partial \sigma(T+K) \\
& \subseteq \operatorname{conv} \sigma_{\text {lre }}(T+K)=\operatorname{conv} \sigma_{\text {lre }}(T) .
\end{aligned}
$$

Hence $W_{e}(T)=W_{e}(T+K) \subseteq$ conv $\sigma_{\text {lre }}(T)$. In view of Lemma 2.7, we obtain $\operatorname{conv} \sigma_{\text {lre }}(T)=W_{e}(T)$.

Given $T \in \mathcal{B}(\mathcal{H})$ and a subspace $M$ of $\mathcal{H}$, we let $T_{M}$ denote the compression of $T$ to $M$. Here subspace means closed linear manifold.

Lemma 2.10 ([7], Thm. 5.1). Let $T \in \mathcal{B}(\mathcal{H})$. If $M$ is a subspace of $\mathcal{H}$ with $\operatorname{dim} M^{\perp}<\infty$, then $W_{e}\left(T_{M}\right)=W_{e}(T)$.

Lemma 2.11 ([11], Prop. 4.28). Let $T \in \mathcal{B}(\mathcal{H})$ be hyponormal and $N$ be a normal operator with $\sigma(N) \subseteq \sigma_{\text {lre }}(T)$. Then $T \cong_{a} T \oplus N$. 
Lemma 2.12. Let $T \in \mathcal{B}(\mathcal{H})$. If $\lambda \in \sigma_{\text {lre }}(T)$ and $\|T\|=|\lambda|$, then there exists $K \in \mathcal{K}(\mathcal{H})$ such that $T+K=\lambda I \oplus A$, where $I$ is the identity operator on some infinite-dimensional subspace $M$ of $\mathcal{H}$ and $A \in \mathcal{B}\left(M^{\perp}\right)$.

Proof. Since $\lambda \in \sigma_{\text {lre }}(T)$, by Lemma 2.7, we have $\lambda \in W_{e}(T)$. It follows from Lemma 2.6 that there exists an orthonormal sequence $\left\{e_{n}\right\}_{n=1}^{\infty}$ such that $\mid\left\langle T e_{n}, e_{n}\right\rangle-$ $\lambda \mid<\frac{1}{2^{n}}$ for all $n \geq 1$. Denote $M=\vee\left\{e_{i}: i \geq 1\right\}$. Then $T$ can be written as

$$
T=\left[\begin{array}{ccccc}
a_{1,1} & a_{1,2} & a_{1,3} & \cdots & B_{1} \\
a_{2,1} & a_{2,2} & a_{2,3} & \cdots & B_{2} \\
a_{3,1} & a_{3,2} & a_{3,3} & \cdots & B_{3} \\
\vdots & \vdots & \vdots & \ddots & \vdots \\
C_{1} & C_{2} & C_{3} & \cdots & A
\end{array}\right] \begin{gathered}
e_{2} \\
e_{3} \\
M^{\perp}
\end{gathered}
$$

where $B_{i}: M^{\perp} \rightarrow \vee\left\{e_{i}\right\}$ and $C_{i}: \vee\left\{e_{i}\right\} \rightarrow M^{\perp}$ are both of rank one for $i \geq 1$. Note that $a_{i, j}=\left\langle T e_{j}, e_{i}\right\rangle$ for all $i, j \geq 1$. Hence $\left|a_{n, n}-\lambda\right|<\frac{1}{2^{n}}$ for all $n \geq 1$.

Now fix an $n \geq 1$. Then it is obvious that

$$
\left\{\left\|B_{n}\right\|^{2}+\left|a_{n, n}\right|^{2}+\sum_{i=n+1}^{\infty}\left|a_{n, i}\right|^{2}\right\}^{\frac{1}{2}} \leq\|T\|=|\lambda| .
$$

It follows that

$$
\begin{aligned}
\left\|B_{n}\right\|^{2}+\sum_{i=n+1}^{\infty}\left|a_{n, i}\right|^{2} & \leq|\lambda|^{2}-\left|a_{n, n}\right|^{2} \\
& \leq\left(|\lambda|-\left|a_{n, n}\right|\right)\left(|\lambda|+\left|a_{n, n}\right|\right) \\
& \leq\left|\lambda-a_{n, n}\right|\left(2|\lambda|+\left|\lambda-a_{n, n}\right|\right) \\
& \leq\left|\lambda-a_{n, n}\right|(2|\lambda|+1) \\
& <\frac{2|\lambda|+1}{2^{n}} .
\end{aligned}
$$

Similarly one can prove that

$$
\left\|C_{n}\right\|^{2}+\sum_{i=n+1}^{\infty}\left|a_{i, n}\right|^{2} \leq \frac{2|\lambda|+1}{2^{n}}
$$

Thus the following operator

$$
K_{1}=-\left[\begin{array}{ccccc}
0 & a_{1,2} & a_{1,3} & \cdots & B_{1} \\
a_{2,1} & 0 & a_{2,3} & \cdots & B_{2} \\
a_{3,1} & a_{3,2} & 0 & \cdots & B_{3} \\
\vdots & \vdots & \vdots & \ddots & \vdots \\
C_{1} & C_{2} & C_{3} & \cdots & 0
\end{array}\right] \begin{gathered}
e_{3} \\
\vdots \\
M^{\perp}
\end{gathered}
$$

is compact and

$$
T+K_{1}=\left[\begin{array}{llll}
a_{1,1} & & & \\
& a_{2,2} & & \\
& & \ddots & \\
& & & A
\end{array}\right] \begin{gathered}
e_{1} \\
e_{2} \\
\vdots \\
M^{\perp}
\end{gathered}
$$


Noting that $\left|a_{n, n}-\lambda\right| \rightarrow 0$, there exists compact $K_{2} \in \mathcal{K}(\mathcal{H})$ such that

$$
T+K_{1}+K_{2}=\left[\begin{array}{cccc}
\lambda & & & \\
& \lambda & & \\
& & \ddots & \\
& & & A
\end{array}\right] \begin{gathered}
e_{1} \\
e_{2} \\
\vdots \\
M^{\perp}
\end{gathered} .
$$

This completes the proof.

Let $\mathcal{A}$ be a $C^{*}$-algebra and $\rho_{i}$ be a ${ }^{*}$-representation of $\mathcal{A}$ on $\mathcal{H}_{i}(i=1,2) . \quad \rho_{1}$ and $\rho_{2}$ are said to be approximately unitarily equivalent, denoted by $\rho_{1} \cong_{a} \rho_{2}$, if there exist unitary operators $U_{n}: \mathcal{H}_{1} \rightarrow \mathcal{H}_{2}(n=1,2, \cdots)$ such that

$$
U_{n} \rho_{1}(a)-\rho_{2}(a) U_{n} \rightarrow 0, \quad \forall a \in \mathcal{A} .
$$

The following result is Voiculescu's non-commutative Weyl-von Neumann Theorem [19].

Lemma 2.13. If $\mathcal{A}$ is a separable $C^{*}$-subalgebra of $\mathcal{B}(\mathcal{H})$ and $\rho$ is a ${ }^{*}$-representation of $\mathcal{A}$ on $\mathcal{H}_{\rho}$ such that $\mathcal{A} \cap \mathcal{K}(\mathcal{H}) \subseteq \operatorname{ker} \rho$, then $\mathrm{id} \cong{ }_{a} \mathrm{id} \oplus \rho$, where id is the identity representation of $\mathcal{A}$.

\section{Proofs of Theorems 1.1 AND 1.2}

We first prove several auxiliary results.

Theorem 3.1. Let $T \in \mathcal{B}(\mathcal{H})$ be normaloid. If $N$ is a normal operator on some Hilbert space with $\sigma(N) \subseteq\left\{z \in \sigma_{\text {lre }}(T):|z|=\|T\|\right\}$, then $T \cong_{a} T \oplus N$.

Proof. Denote $\Gamma=\left\{z \in \sigma_{\text {lre }}(T):|z|=\|T\|\right\}$. We claim that $T \cong_{a} T \oplus \lambda I$ for any $\lambda \in \Gamma$, where $I$ is the identity operator on $\mathcal{H}$.

Let $\mathcal{A}$ denote the $C^{*}$-subalgebra of $\mathcal{B}(\mathcal{H})$ generated by $T, I$ and all compact operators on $\mathcal{H}$. Then $\mathcal{A}$ is separable. If $\lambda \in \Gamma$, then $|\lambda|=\|T\|$ and, by Lemma 2.12, there exists $K \in \mathcal{K}(\mathcal{H})$ such that $T+K=\lambda I_{1} \oplus A$, where $I_{1}$ is the identity operator on some infinite-dimensional subspace $M$ of $\mathcal{H}$ and $A \in \mathcal{B}\left(M^{\perp}\right)$. So each operator $X$ in $\mathcal{A}$ can be written as

$$
X=\left[\begin{array}{cc}
\alpha I_{1}+K_{1,1} & K_{1,2} \\
K_{2,1} & *
\end{array}\right] \begin{gathered}
M \\
M^{\perp}
\end{gathered}
$$

where $K_{1,1}, K_{1,2}$ and $K_{2,1}$ are all compact. For such $X$, we define $\rho(X)=\alpha I$. It is easy to see that $\rho$ is a unital *-representation of $\mathcal{A}$ on $\mathcal{H}$ and $\mathcal{K}(\mathcal{H}) \subseteq \operatorname{ker} \rho$. By Lemma 2.13, we have id $\cong_{a}$ id $\oplus \rho$. Note that $\rho(T)=\lambda I$. We have $T \cong_{a} T \oplus \lambda I$. This proves the claim.

Since $\sigma(N) \subseteq \Gamma$, it follows that $\Gamma$ is a nonempty compact set. Without loss of generality, we may assume that $\left\{\lambda_{i}: i=1,2, \cdots\right\}$ is a dense subset of $\Gamma$. Then, by the preceding claim, we have

$$
T \cong_{a} T \oplus \lambda_{1} I \cong_{a} T \oplus \lambda_{2} I \oplus \lambda_{1} I \cong_{a} \ldots \cong_{a} T \oplus\left(\oplus_{i=1}^{n} \lambda_{i} I\right)
$$

for each $n \geq 1$. 
Given an $\varepsilon>0,\left\{B\left(\lambda_{i}, \varepsilon\right)\right\}_{i=1}^{\infty}$ is an open cover of $\Gamma$. Here $B\left(\lambda_{i}, \varepsilon\right) \triangleq\{z \in \mathbb{C}$ : $\left.\left|z-\lambda_{i}\right|<\varepsilon\right\}$. Then there exists $k \geq 1$ such that $\left\{B\left(\lambda_{i}, \varepsilon\right)\right\}_{i=1}^{k}$ is an open cover of $\Gamma$. Then there exists $Y \in \mathcal{B}\left(\mathcal{H}^{(\infty)}\right)$ with $\|Y\|<\varepsilon$ such that

$$
T \oplus\left(\oplus_{i=1}^{\infty} \lambda_{i} I\right)+Y \cong T \oplus\left(\oplus_{i=1}^{k} \lambda_{i} I\right) \cong_{a} T .
$$

Here $\cong$ denotes unitary equivalence. It follows that $T \oplus\left(\oplus_{i=1}^{\infty} \lambda_{i} I\right) \cong_{a} T$. Note that

$$
\sigma\left(\oplus_{i=1}^{\infty} \lambda_{i} I\right)=\sigma_{\text {lre }}\left(\oplus_{i=1}^{\infty} \lambda_{i} I\right)=\Gamma .
$$

Since $N$ is a normal operator with $\sigma(N) \subseteq \Gamma$, by Lemma 2.11, we have

$$
N \oplus\left(\oplus_{i=1}^{\infty} \lambda_{i} I\right) \cong_{a} \oplus_{i=1}^{\infty} \lambda_{i} I .
$$

We deduce that

$$
\begin{aligned}
T \oplus N & \cong_{a}\left(T \oplus\left(\oplus_{i=1}^{\infty} \lambda_{i} I\right)\right) \oplus N \\
& \cong_{a} T \oplus\left(\left(\oplus_{i=1}^{\infty} \lambda_{i} I\right) \oplus N\right) \\
& \cong_{a} T \oplus\left(\oplus_{i=1}^{\infty} \lambda_{i} I\right) \cong_{a} T .
\end{aligned}
$$

This completes the proof.

By the latter half of the above proof, one can see the following result.

Corollary 3.2. Let $T \in \mathcal{B}(\mathcal{H})$ and $\Gamma$ be a compact subset of $\mathbb{C}$. If $T \cong_{a} T \oplus \lambda I$ for any $\lambda \in \Gamma$ and $N$ is a normal operator on some Hilbert space with $\sigma(N)=\Gamma$, where $I$ is the identity operator on $\mathcal{H}$, then $T \cong_{a} T \oplus N$.

The following lemma is well known. For the reader's convenience, we write down its proof.

Lemma 3.3. Let $T \in \mathcal{B}(\mathcal{H})$. If $\lambda \in W(T)$ and $|\lambda|=\|T\|$, then $\lambda \in \sigma_{p}(T)$ and $\operatorname{ker}(T-\lambda)$ reduces $T$.

Proof. Since $\lambda \in W(T)$, there exists a unit vector $e \in \mathcal{H}$ such that $\langle T e, e\rangle=\lambda$. Thus $T$ can be written as

$$
T=\left[\begin{array}{cc}
\lambda & E \\
F & G
\end{array}\right]\{e\}^{\perp} .
$$

Since $|\lambda|=\|T\|$, it follows immediately that $E, F$ are both zero. So $\lambda \in \sigma_{p}(T)$.

Arbitrarily choose a unit vector $f \in \operatorname{ker}(T-\lambda)$. Then $T$ admits the following matrix representation

$$
T=\left[\begin{array}{cc}
\lambda & E_{1} \\
0 & G_{1}
\end{array}\right]\left\{\begin{array}{c}
f \\
\{f\}^{\perp}
\end{array} .\right.
$$

Using a similar argument as above, one can deduce that $E_{1}=0$ and hence $\vee\{f\}$ reduces $T$. Since $f \in \operatorname{ker}(T-\lambda)$ is arbitrary, it follows that $\operatorname{ker}(T-\lambda)$ reduces $T$.

Corollary 3.4. Let $T \in \mathcal{B}(\mathcal{H})$ be transaloid. If $\lambda \in \sigma_{0}(T) \cap \mathcal{E}(\operatorname{conv} \sigma(T))$, then $\lambda \in \sigma_{p}(T)$ and $\operatorname{ker}(T-\lambda)$ reduces $T$. 
Proof. Since $\lambda$ is an extreme point of conv $\sigma(T)$, we can find $\mu \in \mathbb{C}$ such that

$$
\begin{aligned}
|\mu-\lambda| & =\max \{|\mu-z|: z \in \operatorname{conv} \sigma(T)\} \\
& =\max \{|\mu-z|: z \in \sigma(T)\} \\
& =\gamma(\mu-T) .
\end{aligned}
$$

Noting that $T$ is transaloid, we have $|\mu-\lambda|=\|\mu-T\|$. On the other hand, since $\lambda \in \sigma_{0}(T) \subseteq \sigma_{p}(T)$, we have $\mu-\lambda \in \sigma_{p}(\mu-T) \subseteq W(\mu-T)$. By Lemma 3.3, $\operatorname{ker}((\mu-T)-(\mu-\lambda))$ reduces $T-\mu$, that is, $\operatorname{ker}(T-\lambda)$ reduces $T$.

Corollary 3.5. Let $T \in \mathcal{B}(\mathcal{H})$. If $\lambda \in \sigma_{0}(T)$ and there exists $z_{0} \in \mathbb{C} \backslash \sigma(T)$ such that

$$
\left|\left(\lambda-z_{0}\right)^{-1}\right|=\left\|\left(T-z_{0}\right)^{-1}\right\|,
$$

then $\lambda \in \sigma_{p}(T)$ and $\operatorname{ker}(T-\lambda)$ reduces $T$.

Proof. Note that

$$
\left(T-z_{0}\right)^{-1}-\left(\lambda-z_{0}\right)^{-1}=\left(T-z_{0}\right)^{-1}(\lambda-T)\left(\lambda-z_{0}\right)^{-1} .
$$

Then $\operatorname{ker}\left(\left(T-z_{0}\right)^{-1}-\left(\lambda-z_{0}\right)^{-1}\right)=\operatorname{ker}(T-\lambda)$. Since $\lambda \in \sigma_{0}(T)$, we have $\lambda \in \sigma_{p}(T)$ and

$$
\left(\lambda-z_{0}\right)^{-1} \in \sigma_{p}\left(\left(T-z_{0}\right)^{-1}\right) \subseteq W\left(\left(T-z_{0}\right)^{-1}\right) .
$$

Then it follows from Lemma 3.3 that $\operatorname{ker}\left(\left(T-z_{0}\right)^{-1}-\left(\lambda-z_{0}\right)^{-1}\right)$ reduces $\left(T-z_{0}\right)^{-1}$. That is, $\operatorname{ker}(T-\lambda)$ reduces $T$.

Now we can give the proof of Theorem 1.1.

Proof of Theorem 1.1. "£". Since $N$ must satisfy $\|N\|=\gamma(N)$, we have

$$
\|T\|=\|N\|=\gamma(N) \leq \gamma(T) \leq\|T\| .
$$

So $\|T\|=\gamma(T)$. This proves the sufficiency.

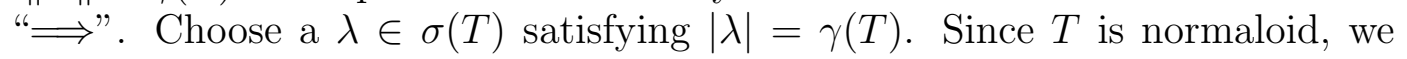
have $|\lambda|=\|T\|$. It is obvious that $\lambda \in \partial \sigma(T)$.

If $\lambda \in \sigma_{\text {lre }}(T)$, then, by Theorem 3.1, $T \cong_{a} \lambda I \oplus T$, as desired.

If $\lambda \notin \sigma_{\text {lre }}(T)$, then, by Lemma 2.1, $\lambda \in \sigma_{0}(T) \subseteq \sigma_{p}(T)$. So $\lambda \in W(T)$. It follows from Lemma 3.3 that $\operatorname{ker}(T-\lambda)$ reduces $T$. Hence $T$ can be written as

$$
T=\left[\begin{array}{cc}
\lambda I_{1} & 0 \\
0 & A
\end{array}\right] \begin{gathered}
M \\
M^{\perp}
\end{gathered}
$$

where $M=\operatorname{ker}(T-\lambda)$ and $I_{1}$ is the identity operator on $M$. This completes the proof.

The rest of this section is devoted to the proof of Theorem 1.2. Still, we need several auxiliary results.

If $T \in \mathcal{B}(\mathcal{H})$ is hyponormal and $N$ is a normal operator with $\sigma(N) \subseteq \sigma_{\text {lre }}(T)$, then $T \cong_{a} T \oplus N$ (see [11, Prop. 4.28]). The following result extends this to certain transaloid operators.

Theorem 3.6. Let $T \in \mathcal{B}(\mathcal{H})$ be transaloid. If $N$ is a normal operator with its spectrum contained in $\sigma_{\text {lre }}(T) \cap \mathcal{E}(\operatorname{conv} \sigma(T))$, then $T \cong_{a} T \oplus N$. 
Proof. Denote $\Gamma=\sigma_{\text {lre }}(T) \cap \mathcal{E}(\operatorname{conv} \sigma(T))$. By Corollary 3.2, it suffices to prove that $T \cong_{a} T \oplus \lambda I$ for any $\lambda \in \Gamma$, where $I$ is the identity operator on $\mathcal{H}$.

Now fix a $\lambda \in \Gamma$. Then it is obvious that $\lambda \in \partial \sigma(T)$. Since $\lambda$ is an extreme point of conv $\sigma(T)$, we can find $\mu \in \mathbb{C}$ such that

$$
\begin{aligned}
|\mu-\lambda| & =\max \{|\mu-z|: z \in \operatorname{conv} \sigma(T)\} \\
& =\max \{|\mu-z|: z \in \sigma(T)\} \\
& =\gamma(\mu-T) .
\end{aligned}
$$

Noting that $T$ is transaloid, we have $|\mu-\lambda|=\|\mu-T\|$. Since $\lambda \in \sigma_{\text {lre }}(T)$ and hence $\mu-\lambda \in \sigma_{\text {lre }}(\mu-T)$, it follows from Theorem 3.1 that $(\mu-T) \cong_{a}(\mu-T) \oplus(\mu-\lambda) I$, that is, $T \cong_{a} T \oplus \lambda I$. By Corollary 3.2, this completes the proof.

Remark 3.7. For a transaloid operator $T$, it can happen that $\sigma_{\text {lre }}(T) \cap \mathcal{E}(\operatorname{conv} \sigma(T))$ $=\emptyset$. In this case, it is possible that there exists no normal operator $N$ satisfying $T \cong_{a} T \oplus N$. In the following we shall give an example of such operator (Example 3.8). This shows that the result of Theorem 3.6 is sharp.

By Lemma 2.9, each hyponormal operator $A$ satisfies $W_{e}(A)=\operatorname{conv} \sigma_{\text {lre }}(A)$. For transaloid operators, this is not the case. Here is an example.

Example 3.8. Let $I$ be the identity operator on $\mathcal{H}$ and set

$$
A=\left[\begin{array}{ll}
0 & I \\
0 & 0
\end{array}\right] \begin{aligned}
& \mathcal{H} \\
& \mathcal{H}
\end{aligned}
$$

Then $\sigma(A)=\sigma_{\text {lre }}(A)=\{0\}$ and $W(A)=W_{e}(A)=\left\{z \in \mathbb{C}:|z| \leq \frac{1}{2}\right\}$. Let $N$ be the diagonal operator $\operatorname{diag}\{2 \mathrm{i}, \sqrt{3}-\mathrm{i},-\sqrt{3}-\mathrm{i}\}$ on $\mathbb{C}^{3}$. Set $T=A \oplus N$. It is obvious that $W_{e}(T)=W_{e}(A)=W(A)$ and $\sigma_{\text {lre }}(T)=\{0\}$. So $W_{e}(T) \neq \operatorname{conv} \sigma_{\text {lre }}(T)$. It remains to check that $T$ is transaloid.

Since the closed unit disk $\mathbb{D}^{-}$is contained in $\Gamma \triangleq \operatorname{conv}\{2 \mathrm{i}, \sqrt{3}-\mathrm{i},-\sqrt{3}-\mathrm{i}\}$, we have

$$
\begin{aligned}
\|A-\lambda\| & \leq 1+|\lambda| \\
& =\max \left\{|u-\lambda|: \mu \in \mathbb{D}^{-}\right\} \\
& \leq \max \{|u-\lambda|: \mu \in \Gamma\} \\
& =\max \{|2 \mathrm{i}-\lambda|,|\sqrt{3}-\mathrm{i}-\lambda|,|-\sqrt{3}-\mathrm{i}-\lambda|\} \\
& =\gamma(N-\lambda)=\|N-\lambda\|, \quad \forall \lambda \in \mathbb{C} .
\end{aligned}
$$

Then $\|T-\lambda\|=\|N-\lambda\|=\gamma(N-\lambda) \leq \gamma(T-\lambda)$. So $T$ is transaloid.

Proposition 3.9. Let $T$ be the transaloid operator defined in Example 3.8. Then there exists no normal operator $P$ such that $T \cong_{a} T \oplus P$.

Proof. For a proof by contradiction, we assume that $P$ is a normal operator on some Hilbert space satisfying $T \cong_{a} T \oplus P$. Thus $\sigma(P) \subseteq \sigma(T \oplus P)=\sigma(T)$. Noting that $\sigma(T)=\{0,2 \mathrm{i}, \sqrt{3}-\mathrm{i},-\sqrt{3}-\mathrm{i}\}$, we may assume that $P=P_{1} \oplus P_{2}$, where $\sigma\left(P_{1}\right) \subseteq\{0\}$ and $\sigma\left(P_{2}\right) \subseteq\{2 \mathrm{i}, \sqrt{3}-\mathrm{i},-\sqrt{3}-\mathrm{i}\}$. This is because $P$ is normal. 
Note that $T=N \oplus A, T \oplus P=\left(N \oplus P_{2}\right) \oplus\left(A \oplus P_{1}\right)$ and

$$
\sigma(N)=\sigma\left(N \oplus P_{2}\right)=\{2 \mathrm{i}, \sqrt{3}-\mathrm{i},-\sqrt{3}-\mathrm{i}\}, \quad \sigma(A)=\sigma\left(A \oplus P_{1}\right)=\{0\} .
$$

Since $T \cong_{a} T \oplus P$, it follows that $N \cong_{a} N \oplus P_{2}$ and $A \cong_{a} A \oplus P_{1}$. Noting that $N$ acts on a finite-dimensional Hilbert space, one can deduce that $P_{2}$ is absent and hence $P=P_{1}$.

One can observe that the $C^{*}$-algebra $C^{*}(A)$ generated by $A$ is ${ }^{*}$-isomorphic to $M_{2}(\mathbb{C})$. Thus $C^{*}(A)$ admits no character on it. However, $C^{*}\left(A \oplus P_{1}\right)$ admits at least one character on it since $P_{1}$ is normal. On the other hand, it follows from $A \cong{ }_{a} A \oplus P_{1}$ that $C^{*}(A)$ is ${ }^{*}$-isomorphic to $C^{*}\left(A \oplus P_{1}\right)$. This is a contradiction, and we complete the proof.

Now we are ready to give the proof of Theorem 1.2.

Proof of Theorem 1.2. Denote $\Gamma=\sigma_{\text {lre }}(T) \cap \mathcal{E}(\operatorname{conv} \sigma(T))$.

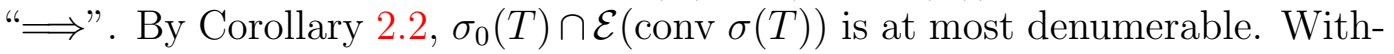
out loss of generality, we assume that $\sigma_{0}(T) \cap \mathcal{E}(\operatorname{conv} \sigma(T))=\left\{\lambda_{i}: i \geq 1\right\}$. By Corollary 3.4, $T$ can be written as $T=N_{1} \oplus A$, where $N_{1}$ is a diagonal normal operator with eigenvalues $\left\{\lambda_{i}: i \geq 1\right\}$ and $\sigma(A) \subseteq \sigma(T)$.

Choose a normal operator $N_{2}$ on $\mathcal{H}$ with $\sigma\left(N_{2}\right)=\sigma_{e}\left(N_{2}\right)=\Gamma$. Then, by Theorem 3.6, $T \cong{ }_{a} T \oplus N_{2}$. Set $N=N_{1} \oplus N_{2}$. Thus $N$ is normal and

$$
T \cong{ }_{a} T \oplus N_{2}=A \oplus N_{1} \oplus N_{2}=A \oplus N .
$$

Since $\mathcal{E}(\operatorname{conv} \sigma(T)) \subseteq \partial \sigma(T)$, it follows from Lemma 2.1 that $\mathcal{E}(\operatorname{conv} \sigma(T))=$ $\Gamma \cup\left\{\lambda_{i}: i \geq 1\right\}$. Then $\sigma(N)=\mathcal{E}(\operatorname{conv} \sigma(T))$. Now it suffices to prove that $\|A-\lambda\| \leq\|N-\lambda\|$ for all $\lambda \in \mathbb{C}$.

For $\lambda \in \mathbb{C}$, since $N, T$ are both transaloid, we have

$$
\begin{aligned}
\|A-\lambda\| & \leq\|T-\lambda\|=\gamma(T-\lambda) \\
& =\max \{|\mu-\lambda|: \mu \in \sigma(T)\} \\
& =\max \{|\mu-\lambda|: \mu \in \mathcal{E}(\operatorname{conv} \sigma(T))\} \\
& =\max \{|\mu-\lambda|: \mu \in \sigma(N)\} \\
& =\gamma(N-\lambda)=\|N-\lambda\| .
\end{aligned}
$$

"£". If two operators $X, Y$ are approximately unitarily equivalent, then it is obvious that $\sigma(X)=\sigma(Y)$ and $\gamma(X)=\gamma(Y)$. For $\lambda \in \mathbb{C}$, since $N$ is transaloid, we have

$$
\begin{aligned}
\|T-\lambda\| & \geq \gamma(T-\lambda) \\
& =\gamma(N \oplus A-\lambda) \\
& \geq \gamma(N-\lambda) \\
& =\|N-\lambda\| \\
& =\max \{\|N-\lambda\|,\|A-\lambda\|\} \\
& =\|N \oplus A-\lambda\|=\|T-\lambda\|,
\end{aligned}
$$

that is, $\gamma(T-\lambda)=\|T-\lambda\|$. So $T$ is transaloid. 


\section{Proofs of Theorems 1.3 AND 1.4}

Lemma 4.1. Let $T \in \mathcal{B}(\mathcal{H})$ be p-normaloid. If $f$ is analytic in an open set containing $\widehat{\sigma(T)}$, then $f(T)$ is normaloid; in particular, $(T-z)^{-1}$ is normaloid for any $z \notin \widehat{\sigma(T)}$.

Proof. Note that $\mathbb{C} \backslash \widehat{\sigma(T)}$ is connected. By the Runge theorem, there exist polynomials $\left\{p_{n}\right\}$ converge to $f$ uniformly on a neighborhood of $\widehat{\sigma(T)}$. Thus, by [11, Prop. 1.7], $p_{n}(T) \rightarrow f(T)$. Since the set of normaloid operators is closed, one can deduce that $f(T)$ is normaloid.

By the Runge theorem, using a similar argument as above, one can see the following corollary.

Corollary 4.2. Let $T \in \mathcal{B}(\mathcal{H})$ be a von Neumann operator. If $f$ is analytic in an open set containing $\sigma(T)$, then $f(T)$ is normaloid; in particular, $(T-z)^{-1}$ is normaloid for any $z \in \mathbb{C} \backslash \sigma(T)$.

Theorem 4.3. Let $T \in \mathcal{B}(\mathcal{H})$ be p-normaloid. If $N$ is a normal operator with its spectrum contained in $\sigma_{\text {lre }}(T) \cap \partial \widehat{\sigma(T)}$, then $T \cong_{a} T \oplus N$.

Proof. Denote $\Gamma=\sigma_{\text {lre }}(T) \cap \partial \widehat{\sigma(T)}$. By Corollary 3.2, it suffices to prove that $T \cong_{a} T \oplus \lambda I$ for any $\lambda \in \Gamma$, where $I$ is the identity operator on $\mathcal{H}$.

Now we fix a $\lambda_{0} \in \Gamma$. Denote by $\Omega$ the unbounded component of $\mathbb{C} \backslash \sigma(T)$. So $\mathbb{C} \backslash \Omega=\widehat{\sigma(T)}$. Since $\lambda_{0} \in \partial \widehat{\sigma(T)}=\partial \Omega$, we can choose $\left\{z_{n}\right\}_{n=1}^{\infty} \subseteq \Omega$ such that $z_{n} \rightarrow$ $\lambda_{0}$. For each $n \geq 1$, we can find $\lambda_{n} \in \sigma(T)$ such that $\operatorname{dist}\left(z_{n}, \sigma(T)\right)=\left|z_{n}-\lambda_{n}\right|$. It is obvious that $\lambda_{n} \in \partial \sigma(T)$ and $\lambda_{n} \rightarrow \lambda_{0}$ since $\operatorname{dist}\left(z_{n}, \sigma(T)\right) \leq\left|z_{n}-\lambda_{0}\right| \rightarrow 0$.

For each $n \geq 1$, since $z_{n} \in \Omega=\mathbb{C} \backslash \widehat{\sigma(T)}$, by Lemma $4.1,\left(T-z_{n}\right)^{-1}$ is normaloid. Then

$$
\begin{aligned}
\left\|\left(T-z_{n}\right)^{-1}\right\| & =\gamma\left(\left(T-z_{n}\right)^{-1}\right)=\max \left\{\frac{1}{\left|z-z_{n}\right|}: z \in \sigma(T)\right\} \\
& =\frac{1}{\operatorname{dist}\left(z_{n}, \sigma(T)\right)}=\frac{1}{\left|\lambda_{n}-z_{n}\right|} .
\end{aligned}
$$

Note that $\frac{1}{\lambda_{n}-z_{n}} \in \sigma\left(\left(T-z_{n}\right)^{-1}\right)$ for $n \geq 1$.

Case 1. There exist $n_{1}<n_{2}<n_{3}<\cdots$ such that $\lambda_{n_{k}} \in \sigma_{\text {lre }}(T)$. If this holds, then $\frac{1}{\lambda_{n_{k}}-z_{n_{k}}} \in \sigma_{\text {lre }}\left(\left(T-z_{n_{k}}\right)^{-1}\right)$. Then by Theorem 3.1 we have

$$
\left(T-z_{n_{k}}\right)^{-1} \cong_{a}\left(T-z_{n_{k}}\right)^{-1} \oplus\left(\lambda_{n_{k}}-z_{n_{k}}\right)^{-1} I, \quad \forall k \geq 1 .
$$

It follows immediately that $T \cong_{a} T \oplus \lambda_{n_{k}} I$ for all $k \geq 1$. Since $\lambda_{n_{k}} \rightarrow \lambda_{0}$, one can see $T \cong_{a} T \oplus \lambda_{0} I$.

Case 2. There exists $m>0$ such that $\lambda_{n} \notin \sigma_{\text {lre }}(T)$ for $n \geq m$. Since $\lambda_{n} \in$ $\partial \sigma(T)$, it follows from Lemma 2.1 that $\lambda_{n} \in \sigma_{0}(T)$ for $n \geq m$. Noting that $\left\|\left(T-z_{n}\right)^{-1}\right\|=\frac{1}{\left|\lambda_{n}-z_{n}\right|}$, it follows from Corollary 3.5 that $\operatorname{ker}\left(T-\lambda_{n}\right)$ reduces $T$ for $n \geq m$. Noting that $\lambda_{n} \rightarrow \lambda_{0} \in \sigma_{\text {lre }}(T)$, we may directly assume that $\left\{\lambda_{n}: n \geq m\right\}$ are pairwise distinct. So $T$ can be written as

$$
T=A \oplus \operatorname{diag}\left\{\lambda_{m}, \lambda_{m+1}, \lambda_{m+2}, \cdots\right\} .
$$


In view of the convergence $\lambda_{n} \rightarrow \lambda_{0}$, one can see

$$
\lambda_{0} \in \sigma_{\text {lre }}\left(\operatorname{diag}\left\{\lambda_{m}, \lambda_{m+1}, \lambda_{m+2}, \cdots\right\}\right) .
$$

Then, by Lemma 2.11, we have

$$
\operatorname{diag}\left\{\lambda_{m}, \lambda_{m+1}, \lambda_{m+2}, \cdots\right\} \cong_{a} \operatorname{diag}\left\{\lambda_{m}, \lambda_{m+1}, \lambda_{m+2}, \cdots\right\} \oplus \lambda_{0} I
$$

and hence

$$
\begin{aligned}
T & =A \oplus \operatorname{diag}\left\{\lambda_{m}, \lambda_{m+1}, \lambda_{m+2}, \cdots\right\} \\
& \cong{ }_{a} A \oplus\left(\operatorname{diag}\left\{\lambda_{m}, \lambda_{m+1}, \lambda_{m+2}, \cdots\right\} \oplus \lambda_{0} I\right) \\
& \cong\left(A \oplus \operatorname{diag}\left\{\lambda_{m}, \lambda_{m+1}, \lambda_{m+2}, \cdots\right\}\right) \oplus \lambda_{0} I \\
& =T \oplus \lambda_{0} I .
\end{aligned}
$$

Thus we complete the proof in either case.

Using Corollary 4.2 and a similar argument as in the proof of Theorem 4.3, one can prove the following result. We omit its proof.

Theorem 4.4. Let $T \in \mathcal{B}(\mathcal{H})$ be a von Neumann operator. If $N$ is a normal operator with its spectrum contained in $\sigma_{\text {lre }}(T) \cap \partial \sigma(T)$, then $T \cong_{a} T \oplus N$.

Now we can give the proof of Theorem 1.3.

Proof of Theorem 1.3. "£". Since each normal operator is normaloid, for each polynomial $p$, we have

$$
\|p(T)\|=\|p(N)\|=\gamma(p(N)) \leq \gamma(p(T)) \leq\|p(T)\| .
$$

Thus $T$ is p-normaloid.

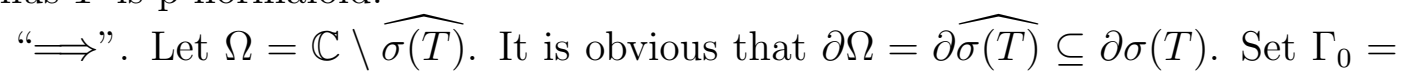
$\partial \Omega \cap \sigma_{0}(T)$ and $\Gamma_{1}=\partial \Omega \cap \sigma_{\text {lre }}(T)$. By Lemma 2.1, we have $\partial \Omega=\Gamma_{0} \cup \Gamma_{1}$. Since $\sigma_{0}(T)$ is at most denumerable, we may directly assume that $\Gamma_{0}=\left\{\lambda_{1}, \lambda_{2}, \lambda_{3}, \cdots\right\}$.

For each $n \geq 1$, since $\lambda_{n} \in \sigma_{0}(T) \cap \partial \Omega$, we can find $z_{n} \in \Omega$ such that $\left|z_{n}-\lambda_{n}\right|<$ $\operatorname{dist}\left(z_{n}, \sigma(T) \backslash\left\{\lambda_{n}\right\}\right)$. Then

$$
\begin{aligned}
\gamma\left(\left(T-z_{n}\right)^{-1}\right) & =\max \left\{\left|\left(\lambda-z_{n}\right)^{-1}\right|: \lambda \in \sigma(T)\right\} \\
& =\frac{1}{\operatorname{dist}\left(z_{n}, \sigma(T)\right)}=\frac{1}{\left|\lambda_{n}-z_{n}\right|} .
\end{aligned}
$$

On the other hand, since $T$ is p-normaloid, it follows from Lemma 4.1 that ( $T$ $\left.z_{n}\right)^{-1}$ is normaloid. Hence

$$
\left\|\left(T-z_{n}\right)^{-1}\right\|=\gamma\left(\left(T-z_{n}\right)^{-1}\right)=\frac{1}{\left|\lambda_{n}-z_{n}\right|} .
$$

Since $\lambda_{n} \in \sigma_{0}(T)$, it follows from Corollary 3.5 that $\operatorname{ker}\left(T-\lambda_{n}\right)$ reduces $T$. It is obvious that $\left\{\lambda_{n}: n \geq 1\right\}$ are pairwise distinct. Then $T$ can be written as

$$
T=A \oplus \operatorname{diag}\left\{\lambda_{1}, \lambda_{2}, \lambda_{3}, \cdots\right\} \text {. }
$$


Choose a normal operator $N_{1}$ on $\mathcal{H}$ with $\sigma\left(N_{1}\right)=\Gamma_{1}$. Then, by Theorem 4.3, we have $T \cong_{a} T \oplus N_{1}$. It follows that

$$
T \cong_{a} T \oplus N_{1}=A \oplus \operatorname{diag}\left\{\lambda_{1}, \lambda_{2}, \lambda_{3}, \cdots\right\} \oplus N_{1} .
$$

Set $N=\operatorname{diag}\left\{\lambda_{1}, \lambda_{2}, \lambda_{3}, \cdots\right\} \oplus N_{1}$. Then $N$ is normal. Since $\Gamma_{1} \cup\left\{\lambda_{n}: n \geq 1\right\}=$ $\partial \Omega$ is compact, one can deduce that $\left\{\lambda_{n}: n \geq 1\right\}^{-} \subseteq \partial \Omega$ and

$$
\sigma(N)=\sigma\left(N_{1}\right) \cup\left\{\lambda_{n}: n \geq 1\right\}^{-}=\partial \Omega=\partial \widehat{\sigma(T)} .
$$

It remains to check that $\|p(N)\| \geq\|p(A)\|$ for each polynomial $p$. Note that

$$
\begin{aligned}
& \|p(A)\| \leq\|p(T)\|=\gamma(p(T)) \quad \text { by (4.1) } \\
& =\max \{|p(z)|: z \in \sigma(T)\} \\
& \leq \max \{|p(z)|: z \in \widehat{\sigma(T)}\} \quad \text { by MMT } \\
& =\max \{|p(z)|: z \in \partial \widehat{\sigma(T)}\} \\
& =\max \{|p(z)|: z \in \sigma(N)\} \\
& =\gamma(p(N))=\|p(N)\|,
\end{aligned}
$$

that is, $\|p(A)\| \leq\|p(N)\|$. This completes the proof.

The proof of Theorem 1.4 follows similar lines as that of Theorem 1.3.

Proof of Theorem 1.4. Since each normal operator is a von Neumann operator, the sufficiency is clear. We need only prove the necessity.

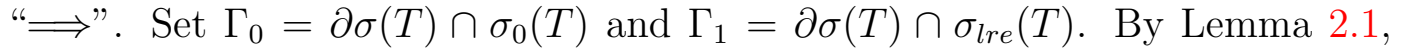
we have $\partial \sigma(T)=\Gamma_{0} \cup \Gamma_{1}$. Since $\sigma_{0}(T)$ is at most denumerable, without loss of generality, we assume that $\Gamma_{0}=\left\{\lambda_{1}, \lambda_{2}, \lambda_{3}, \cdots\right\}$.

For each $n \geq 1$, since $\lambda_{n} \in \sigma_{0}(T)$, we can find $z_{n} \in \mathbb{C} \backslash \sigma(T)$ such that $\left|z_{n}-\lambda_{n}\right|<\operatorname{dist}\left(z_{n}, \sigma(T) \backslash\left\{\lambda_{n}\right\}\right)$. Then

$$
\begin{aligned}
\gamma\left(\left(T-z_{n}\right)^{-1}\right) & =\max \left\{\left|\left(\lambda-z_{n}\right)^{-1}\right|: \lambda \in \sigma(T)\right\} \\
& =\frac{1}{\operatorname{dist}\left(z_{n}, \sigma(T)\right)}=\frac{1}{\left|\lambda_{n}-z_{n}\right|} .
\end{aligned}
$$

On the other hand, since $T$ is a von Neumann operator, it follows from Corollary 4.2 that $\left(T-z_{n}\right)^{-1}$ is normaloid and hence

$$
\left\|\left(T-z_{n}\right)^{-1}\right\|=\gamma\left(\left(T-z_{n}\right)^{-1}\right)=\frac{1}{\left|\lambda_{n}-z_{n}\right|} .
$$

Since $\lambda_{n} \in \sigma_{0}(T)$, it follows from Corollary 3.5 that $\operatorname{ker}\left(T-\lambda_{n}\right)$ reduces $T$. Noting that $\left\{\lambda_{n}: n \geq 1\right\}$ are pairwise distinct, $T$ can be written as

$$
T=A \oplus \operatorname{diag}\left\{\lambda_{1}, \lambda_{2}, \lambda_{3}, \cdots\right\} .
$$

Choose a normal operator $N_{1}$ on $\mathcal{H}$ with $\sigma\left(N_{1}\right)=\Gamma_{1}$. Then, by Theorem 4.4, we have $T \cong_{a} T \oplus N_{1}$. It follows that

$$
T \cong_{a} T \oplus N_{1}=A \oplus \operatorname{diag}\left\{\lambda_{1}, \lambda_{2}, \lambda_{3}, \cdots\right\} \oplus N_{1} .
$$


Set $N=\operatorname{diag}\left\{\lambda_{1}, \lambda_{2}, \lambda_{3}, \cdots\right\} \oplus N_{1}$. Then $N$ is normal and

$$
\sigma(N)=\sigma\left(N_{1}\right) \cup\left\{\lambda_{n}: n \geq 1\right\}=\partial \sigma(T) .
$$

It remains to check that $\|f(N)\| \geq\|f(A)\|$ for each rational function $f$ with poles off $\sigma(T)$. Note that

$$
\begin{aligned}
\|f(A)\| & \leq\|f(T)\|=\gamma(f(T)) \\
& =\max \{|f(z)|: z \in \sigma(T)\} \\
& =\max \{|f(z)|: z \in \partial \sigma(T)\} \\
& =\max \{|f(z)|: z \in \sigma(N)\} \\
& =\gamma(f(N))=\|f(N)\|,
\end{aligned}
$$

that is, $\|f(A)\| \leq\|f(N)\|$. This completes the proof.

Example 4.5. Let $A \in \mathcal{B}(\mathcal{H})$. Using $A$, we shall construct several normaloid operators. Denote $r=\|A\|$. Set $N_{1}=r \in \mathcal{B}(\mathbb{C})$ and $N_{2}=\operatorname{diag}\{2 r \mathrm{i},(\sqrt{3}-$ i) $r,-(\sqrt{3}+\mathrm{i}) r\} \in \mathcal{B}\left(\mathbb{C}^{3}\right)$. Let $N_{3}$ be a normal operator with $\sigma\left(N_{3}\right)=\{z \in \mathbb{C}$ : $|z|=r\}$ and $N_{4}$ be a normal operator with $\sigma\left(N_{4}\right)=\{z \in \mathbb{C}:|z| \leq r\}$.

For $1 \leq i \leq 4$, set $T_{i}=A \oplus N_{i}$. Compute to see that $\left\|N_{1}\right\|=r=\|A\|$ and

$$
\begin{aligned}
\|A-\lambda\| & \leq\|A\|+|\lambda|=r+|\lambda| \\
& =\max \{|\lambda-z|:|z|=r\} \\
& \leq \max \{|\lambda-2 r \mathrm{i}|,|\lambda-(\sqrt{3}-\mathrm{i}) r|,|\lambda+(\sqrt{3}+\mathrm{i}) r|\} \\
& =\gamma\left(N_{2}-\lambda\right)=\left\|N_{2}-\lambda\right\|, \quad \forall \lambda \in \mathbb{C} .
\end{aligned}
$$

By Theorems 1.1 and 1.2, $T_{1}$ is normaloid and $T_{2}$ is transaloid.

For each polynomial $p$, using the von Neumann inequality ([16]), we have

$$
\begin{aligned}
\|p(A)\| & \leq \max \{|p(z)|:|z| \leq r\} \\
& =\max \{|p(z)|:|z|=r\} \\
& =\gamma\left(p\left(N_{3}\right)\right)=\left\|p\left(N_{3}\right)\right\| .
\end{aligned}
$$

By Theorem 1.3, $T_{3}$ is p-normaloid.

Note that $\sigma\left(T_{4}\right)=\{z \in \mathbb{C}:|z| \leq r\}$. For each rational function $f$ with poles off $\sigma\left(T_{4}\right)$, using the von Neumann inequality again, we have

$$
\begin{aligned}
\|f(A)\| & \leq \max \{|f(z)|:|z| \leq r\} \\
& =\gamma\left(f\left(N_{4}\right)\right)=\left\|f\left(N_{4}\right)\right\| .
\end{aligned}
$$

By Theorem 1.4, $T_{4}$ is a von Neumann operator.

Note that the above-mentioned operator $A$ can be arbitrary. Thus one can construct various normaloid operators with prescribed properties.

The rest of this section is devoted to describing when a unilateral weighted shift is a von Neumann operator.

Theorem 4.6. Let $T \in \mathcal{B}(\mathcal{H})$ be a unilateral weighted shift with weights $\left\{w_{i}\right\}_{i=1}^{\infty}$. Then the following are equivalent:

(i) $T$ is normaloid; 
(ii) $T$ is transaloid;

(iii) $T$ is p-normaloid;

(iv) $T$ is a von Neumann operator;

(v) $\sup _{n \geq 1}\left|w_{n}\right|=\lim _{k} \sup _{i \geq 0}\left|w_{i+1} w_{i+2} \cdots w_{i+k}\right|^{1 / k}$.

Proof. Since "(iv) $\Longrightarrow$ (iii) $\Longrightarrow$ (ii) $\Longrightarrow($ i $) \Longleftrightarrow(v)$ " are obvious, we need only prove that "(i) $\Longrightarrow$ (iv)".

Assume that $T$ is normaloid. Then $\gamma(T)=\|T\|$. Since the spectrum of each weighted shift has circular symmetry, it follows from [11, Thm. 3.40] that $\sigma(T)=$ $\{z \in \mathbb{C}:|z| \leq\|T\|\}$. In view of Lemma 2.1, one can deduce that $\partial \sigma(T)=\{z \in$ $\mathbb{C}:|z|=\|T\|\} \subseteq \sigma_{\text {lre }}(T)$. Choose a normal operator $N$ on $\mathcal{H}$ with $\sigma(N)=\{z \in$ $\mathbb{C}:|z|=\|T\|\}$. Then $\sigma(N) \subseteq \sigma_{\text {lre }}(T)$ and, by Theorem 3.1, we have $T \cong_{a} T \oplus N$.

Given a rational function $f$ with poles off $\sigma(T)$, it follows from the von Neumann inequality that

$$
\begin{aligned}
\|f(T)\| & \leq \max \{|f(z)|:|z| \leq\|T\|\} \\
& =\max \{|f(z)|:|z|=\|T\|\} \\
& =\gamma(f(N))=\|f(N)\| .
\end{aligned}
$$

By Theorem 1.4, $T$ is a von Neumann operator.

From the above proof, one can see the following result.

Corollary 4.7. Let $T \in \mathcal{B}(\mathcal{H})$. If $\sigma(T)=\{z \in \mathbb{C}:|z| \leq \delta\}$ for some $\delta \geq 0$, then $T$ is normaloid if and only if $T$ is a von Neumann operator.

\section{Proofs of Theorems 1.7 And 1.9}

We first give an auxiliary result.

Proposition 5.1. Let $T \in \mathcal{B}(\mathcal{H})$ be normal. Then, given $\varepsilon>0$, there exists $K \in \mathcal{K}(\mathcal{H})$ with $\|K\|<\varepsilon$ such that $T+K$ is normal and

$$
W(T)^{-} \subseteq W(T+K)=\operatorname{conv} \sigma(T+K) .
$$

Proof. Fix an $\varepsilon>0$. Let $\Gamma=\mathcal{E}\left(\operatorname{conv} \sigma_{\text {lre }}(T)\right)$. It is obvious that $\Gamma \subseteq \sigma_{\text {lre }}(T)$ is a nonempty compact set. For given $\varepsilon>0$, we can find $a_{1}, a_{2}, \cdots, a_{n} \in \Gamma$ such that

$$
\Gamma \subseteq \bigcup_{i=1}^{n} B\left(a_{i}, \frac{\varepsilon}{3}\right) .
$$

For each $1 \leq i \leq n$, we can choose complex numbers $\mu_{3 i}, \mu_{3 i-1}, \mu_{3 i-2}$ with

$$
\left|a_{i}-\mu_{3 i}\right|=\left|a_{i}-\mu_{3 i-1}\right|=\left|a_{i}-\mu_{3 i-2}\right|=\frac{2 \varepsilon}{3}
$$

such that $B\left(a_{i}, \frac{\varepsilon}{3}\right) \subseteq \operatorname{conv}\left\{\mu_{3 i}, \mu_{3 i-1}, \mu_{3 i-2}\right\}$. For $1 \leq i \leq n$, set $\lambda_{3 i}=\lambda_{3 i-1}=$ $\lambda_{3 i-2}=a_{i}$.

Since each $\lambda_{i}$ lies in $\sigma_{\text {lre }}(T)$, it follows from Lemma 2.11 that

$$
T \cong_{a} T \oplus \operatorname{diag}\left\{\lambda_{1}, \lambda_{2}, \cdots, \lambda_{3 n}\right\} .
$$


Then, by [11, Prop. 4.21 (iv)], there exists $K_{1} \in \mathcal{K}(\mathcal{H})$ with $\left\|K_{1}\right\|<\frac{\varepsilon}{3}$ such that

$$
T+K_{1} \cong\left[\begin{array}{cccc}
\lambda_{1} & & & \\
& \ddots & & \\
& & \lambda_{3 n} & \\
& & & T
\end{array}\right] \begin{gathered}
e_{1} \\
\vdots \\
e_{3 n} \\
\mathcal{H}
\end{gathered}
$$

where $\left\{e_{i}: 1 \leq i \leq 3 n\right\}$ is an orthonormal basis of $\mathbb{C}^{3 n}$. Note that $\left|\lambda_{i}-\mu_{i}\right|=\frac{2 \varepsilon}{3}$ for each $1 \leq i \leq 3 n$. There exists $K_{2} \in \mathcal{K}(\mathcal{H})$ with $\left\|K_{2}\right\|=\frac{2 \varepsilon}{3}$ such that

$$
T+K_{1}+K_{2} \cong\left[\begin{array}{cccc}
\mu_{1} & & & \\
& \ddots & & \\
& & \mu_{3 n} & \\
& & & T
\end{array}\right] \triangleq R .
$$

Set $K=K_{1}+K_{2}$. Then $K \in \mathcal{K}(\mathcal{H})$ and $\|K\|<\varepsilon$. Also $R \cong T+K$ are normal and $W(T) \subseteq W(R)=W(T+K)$. So it remains to check that conv $\sigma(R)=W(R)$. Since $R$ is still normal, by Lemma 2.8, we have $W(R)^{-}=$conv $\sigma(R)$. So it suffices to prove $W(R)=W(R)^{-}$. By Theorem 2.5, we need only check $W_{e}(R) \subseteq W(R)$.

By Lemmas 2.9 and 2.10, we have

$$
\begin{aligned}
W_{e}(R) & =W_{e}(T)=\operatorname{conv} \sigma_{\text {lre }}(T)=\operatorname{conv} \Gamma \\
& \subseteq \operatorname{conv}\left(\cup_{i=1}^{n} B\left(a_{i}, \frac{\varepsilon}{3}\right)\right) \\
& \subseteq \operatorname{conv}\left\{\mu_{i}: 1 \leq i \leq 3 n\right\} \\
& \subseteq W(R) .
\end{aligned}
$$

This completes the proof.

Now we are going to give the proofs of Theorems 1.7 and 1.9.

Proof of Theorem 1.7. By Theorem 1.2, $T$ is approximately unitarily equivalent to an operator with the form $N \oplus A$, where $N$ is normal and

$$
\|A-\lambda\| \leq\|N-\lambda\|, \quad \forall \lambda \in \mathbb{C} .
$$

Without loss of generality, we may directly assume that $T=N \oplus A$, where $N \in \mathcal{B}\left(\mathcal{H}_{1}\right), A \in \mathcal{B}\left(\mathcal{H}_{2}\right)$ and $\mathcal{H}=\mathcal{H}_{1} \oplus \mathcal{H}_{2}$.

Claim. $W(A) \subseteq W(N)^{-}$.

In fact, if not, then, by the convexity of $W(N)^{-}$and $W(A)$, we can find some $\lambda \in \mathbb{C}$ such that

$$
\sup \{|\mu-\lambda|: \mu \in W(A)\}>\sup \left\{|\mu-\lambda|: \mu \in W(N)^{-}\right\},
$$

that is, $\|A-\lambda\| \geq w(A-\lambda)>w(N-\lambda)=\|N-\lambda\|$. This contradicts the hypothesis. So we have proved the claim.

By Proposition 5.1, for any $\varepsilon>0$, we can choose a compact operator $K_{1}$ on $\mathcal{H}_{1}$ with $\left\|K_{1}\right\|<\varepsilon$ such that $N+K_{1}$ is normal and

$$
W(N)^{-} \subseteq W\left(N+K_{1}\right)=\operatorname{conv} \sigma\left(N+K_{1}\right) .
$$


Set

Then $K \in \mathcal{K}(\mathcal{H}),\|K\|<\varepsilon$ and

$$
K=\left[\begin{array}{cc}
K_{1} & 0 \\
0 & 0
\end{array}\right] \mathcal{H}_{1}
$$

$$
T+K=\left[\begin{array}{cc}
N+K_{1} & 0 \\
0 & A
\end{array}\right] \begin{aligned}
& \mathcal{H}_{1} \\
& \mathcal{H}_{2}
\end{aligned}
$$

In view of Claim and (5.2), we obtain

$$
\begin{aligned}
W(T+K) & =\operatorname{conv}\left(W\left(N+K_{1}\right) \cup W(A)\right) \\
& =W\left(N+K_{1}\right) \\
& =\operatorname{conv} \sigma\left(N+K_{1}\right) \\
& \subseteq \operatorname{conv} \sigma(T+K) .
\end{aligned}
$$

So $W(T+K)$ is closed. By [9, Prob. 214], conv $\sigma(T+K) \subseteq W(T+K)^{-}$. Hence conv $\sigma(T+K)=W(T+K)$.

Now fix a $\lambda \in \mathbb{C}$. Since $N+K_{1}$ is normal, it follows from Lemma 2.8 that $w\left(N+K_{1}-\lambda\right)=\left\|N+K_{1}-\lambda\right\|$. Thus

$$
\begin{aligned}
\|A-\lambda\| & \leq\|N-\lambda\| & & \text { by }(5.1) \\
& =w(N-\lambda) & & \text { by }(5.2) \\
& \leq w\left(N+K_{1}-\lambda\right) & & \\
& =\left\|N+K_{1}-\lambda\right\| . & &
\end{aligned}
$$

Noting that $N+K_{1}$ is normal, it follows from Theorem 1.2 that $T+K$ is transaloid. This completes the proof.

Proof of Theorem 1.9. Denote $\Gamma=\mathcal{E}(\operatorname{conv} \sigma(T))$. Then $\Gamma \subseteq \partial \sigma(T)$.

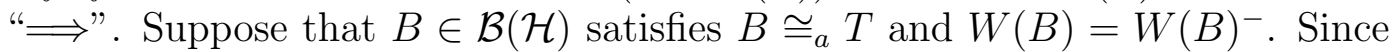
$T$ is transaloid, $B \cong \cong_{a} T$ implies that $B$ is transaloid and $\sigma(B)=\sigma(T)$. Then $\Gamma=\mathcal{E}(\operatorname{conv} \sigma(B))$ and it follows from Lemma 2.8 that conv $\sigma(B)=W(B)$.

We claim that each $\lambda \in \Gamma$ is an eigenvalue of $B$ and $\operatorname{ker}(B-\lambda)$ reduces $B$. Assuming this claim, it follows that $\operatorname{ker}\left(B-\lambda_{1}\right)$ is orthogonal to $\operatorname{ker}\left(B-\lambda_{2}\right)$ for distinct $\lambda_{1}, \lambda_{2} \in \Gamma$. Since $\mathcal{H}$ is separable, one can see that $\Gamma$ is at most denumerable.

Fix a $\lambda \in \Gamma$. Then $\lambda \in \sigma(B) \subseteq W(B)$. Noting that $\lambda$ is an extreme point of conv $\sigma(B)$, there exists $\mu \in \mathbb{C}$ such that

$$
|\lambda-\mu|=\max \{|z-\mu|: z \in \sigma(B)\} .
$$

That is, $|\lambda-\mu|=\gamma(B-\mu)$. Since $B$ is transaloid, we have $|\lambda-\mu|=\|B-\mu\|$. Noting that $\lambda-\mu \in W(B-\mu)$, it follows from Lemma 3.3 that $\lambda-\mu \in \sigma_{p}(B-\mu)$ and $\operatorname{ker}(B-\lambda)$ reduces $B-\lambda$. That is, $\lambda \in \sigma_{p}(B)$ and $\operatorname{ker}(B-\lambda)$ reduces $B$. This proves the necessity.

"£". Since $\partial \sigma(T) \subseteq \sigma_{0}(T) \cup \sigma_{l r e}(T)$ and $\Gamma$ is at most denumerable, without loss of generality, we may assume that

$$
\Gamma \cap \sigma_{0}(T)=\left\{\alpha_{i}: i \geq 1\right\}, \quad \Gamma \cap \sigma_{\text {lre }}(T)=\left\{\beta_{i}: i \geq 1\right\} .
$$

So $\Gamma=\left\{\alpha_{i}, \beta_{i}: i \geq 1\right\}$. 
By Corollary 3.4, $T$ can be written as

$$
T=\operatorname{diag}\left\{\alpha_{1}, \alpha_{2}, \alpha_{3}, \cdots\right\} \oplus A .
$$

It is obvious that $\sigma(A) \subseteq \sigma(T)$.

On the other hand, since each $\beta_{i}$ lies in $\sigma_{\text {lre }}(T) \cap \mathcal{E}(\operatorname{conv} \sigma(T))$, it follows from Theorem 3.6 that

$$
\begin{aligned}
T & \cong_{a} T \oplus \operatorname{diag}\left\{\beta_{1}, \beta_{2}, \beta_{3}, \cdots\right\} \\
& =A \oplus \operatorname{diag}\left\{\alpha_{1}, \alpha_{2}, \alpha_{3}, \cdots\right\} \oplus \operatorname{diag}\left\{\beta_{1}, \beta_{2}, \beta_{3}, \cdots\right\} \triangleq C .
\end{aligned}
$$

Now it remains to check that $W(C)=W(C)^{-}$.

Note that $C$ is transaloid. Then, by Lemma 2.8, we have

$$
\begin{array}{rlr}
W(C)^{-} & =\operatorname{conv} \sigma(C) & \\
& =\operatorname{conv} \sigma(T) & \text { since } T \cong_{a} C \\
& =\operatorname{conv} \Gamma & \\
& =\operatorname{conv}\left\{\alpha_{i}, \beta_{i}: i \geq 1\right\} & \\
& \subseteq W(C) . & \text { since } \alpha_{i}, \beta_{i} \in \sigma_{p}(C)
\end{array}
$$

This completes the proof.

Acknowledgement. The author would like to thank the referee for his/her careful reading and valuable comments. The author acknowledges partial support of National Natural Science Foundation of China (11101177, 11271150).

\section{REFERENCES}

1. P. Aiena, Fredholm and local spectral theory, with applications to multipliers, Kluwer Academic Publishers, Dordrecht, 2004.

2. F.V. Atkinson, The polynomial-normaloid property for Banach-space operators, Monatsh. Math. 79 (1975), no. 4, 273-283.

3. J.-C. Bourin, Compressions and pinchings, J. Operator Theory 50 (2003), no. 2, 211-220.

4. J.B. Conway, A course in functional analysis, Springer-Verlag, New York, 1990.

5. J.B. Conway, The theory of subnormal operators, Mathematical Surveys and Monographs, 36, American Mathematical Society, Providence, RI, 1991.

6. R.E. Curto and Y.M. Han, Weyl's theorem, a-Weyl's theorem, and local spectral theory, J. London Math. Soc. (2) 67 (2003), no. 2, 499-509.

7. P.A. Fillmore, J.G. Stampfli, and J.P. Williams, On the essential numerical range, the essential spectrum, and a problem of Halmos, Acta Sci. Math. (Szeged) 33 (1972), 179192.

8. T. Furuta, Invitation to linear operators: From matrices to bounded linear operators on a Hilbert space, Taylor \& Francis, Ltd., London, 2001.

9. P.R. Halmos, A Hilbert space problem book, 2nd edn. Springer, New York, 1982.

10. F. Hausdorff, Der Wertvorrat einer Bilinearform, Math. Z. 3 (1919), no. 1, 314-316.

11. D.A. Herrero, Approximation of Hilbert space operators, Vol.1. Second ed., Pitman Res. Notes Math. Ser., vol. 224, Longman Sci. Tech., Harlow, 1989.

12. V.I. Istrățescu, On a class of normaloid operators, Math. Z. 124 (1972), 199-202.

13. V.I. Istrăţescu, Introduction to linear operator theory, Monographs and Textbooks in Pure and Applied Mathematics, 65. Marcel Dekker, Inc., New York, 1981.

14. J.S. Lancaster, The boundary of the numerical range, Proc. Amer. Math. Soc. 49 (1975), no. 2, 393-398. 
15. C.S. Lin, Boundary points of the numerical range of an operator, Canad. Math. Bull. 17 (1974/75), no. 5, 689-692.

16. J. von Neumann, Eine Spektraltheorie für allgemeine Operatoren eines unitären Raumes, Math. Nachr. 4 (1951), 258-281.

17. M.H. Stone, Linear transformations in Hilbert space, Reprint of the 1932 original, American Mathematical Society Colloquium Publications, 15, American Mathematical Society, Providence, RI, 1990.

18. O. Toeplitz, Das algebraische Analogon zu einem Satze von Fejér, Math. Z. 2 (1918), no. 1-2, 187-197.

19. D. Voiculescu, A non-commutative Weyl-von Neumann theorem, Rev. Roumaine Math. Pures Appl. 21 (1976), 97-113.

20. A. Wintner, Zur theorie der beschränkten bilinearformen, Math. Z. 30 (1929), no. 1, 228281.

21. S. Zhu and C.G. Li, Operators similar to normaloid operators, J. Korean Math. Soc. 48 (2011), no. 6, 1203-1223.

Department of Mathematics, Jilin University, Changchun 130012, P. R. China. E-mail address: zhusen@jlu.edu.cn 